\title{
Occupations insulaires au Néolithique récent : Groah Denn 1 à Hoëdic (Morbihan)
}

\section{Audrey Blanchard}

\section{(2) OpenEdition}

1 Journals

\section{Édition électronique}

URL : http://journals.openedition.org/abpo/2320

DOI : $10.4000 / a b p o .2320$

ISBN : 978-2-7535-1852-0

ISSN : 2108-6443

\section{Éditeur}

Presses universitaires de Rennes

Édition imprimée

Date de publication : 30 mars 2012

Pagination : 7-30

ISBN : 978-2-7535-1850-6

ISSN : 0399-0826

\section{Référence électronique}




\title{
Occupations insulaires au Néolithique récent : Groah Denn 1 à Hoëdic (Morbihan)
}

\author{
Audrey BLANCHARD \\ Doctorante en archéologie, \\ Université de Rennes I - UMR 6566, CReAAH
}

Le Massif armoricain constitue une exception dans l'histoire de la recherche archéologique. Très tôt les monuments mégalithiques encore en élévation, intriguent voyageurs, antiquaires et collectionneurs. Cette curiosité donne lieu, dès le milieu du XIX ${ }^{\mathrm{e}}$ siècle, à de véritables explorations archéologiques. Toute une série de sites et de monuments du littoral sud breton sont tour à tour inventoriés, prospectés et sondés. Les îles, parmi lesquelles Hoëdic, n'échappent pas à cet intérêt naissant.

Un premier inventaire des sites archéologiques de Houat et Hoëdic est ainsi proposé en 1886 par l'abbé Lavenot alors recteur de l'île d'Hoëdic. Ces observations sont complétées par Z. Le Rouzic, M et St.-J. Péquart lors de leurs visites en 1924. Certains des sites pré- et proto-historiques alors reconnus vont rapidement être explorés, tels l'îlot d'Er Yoh, à l'est de l'île de Houat, en 1924, ou encore la nécropole mésolithique de Port-Neuf sur Hoëdic, dans les années 1930. Durant la seconde moitié du Xx ${ }^{e}$ siècle, les recherches se cantonnent à des prospections ponctuelles. Il faut attendre la fin des années 1990 et le début du Xxi ${ }^{\mathrm{e}}$ siècle pour que la question de l'évolution des peuplements insulaires sur le littoral sud armoricain soit relancée. De nouveaux programmes de recherches (prospections et fouilles archéologiques) émergent alors.

L'île d'Hoëdic fait l'objet au début des années 2000 d'une prospection diachronique, à caractère exhaustif. Dans la continuité de ce recensement, différentes campagnes de fouilles sont entamées parmi lesquelles celles de l'alignement du Douet ${ }^{1}$ et de Groah Denn (dir. J.-M. Large). Les problématiques développées tournent autour de la mise en place d'alignements

1. LARGE, Jean-Marc, Mens, Emmanuel, "L'alignement du Douet à Hoëdic (Morbihan, France) ", L'Anthropologie, n 112, 2008, p. 544-571. 
mégalithiques en contexte îlien. La question d'un particularisme insulaire, tant dans le domaine structurel que la culture matérielle, est rapidement posée.

\section{Le site de Groah Denn}

Lors de l'édification de ces files de blocs, Hoëdic est déjà un territoire limité par la mer de toute part. Toutefois, l'existence d'un passage vers sa voisine Houat, dont elle n'est distante que de 5 kilomètres, n'est pas exclue, notamment lors des plus basses mers. Cette île offre un terrain réduit, ne dépassant pas les 209 hectares (2500 mètres de longueur pour 800 mètres de largeur) pour une altitude maximale de 22 mètres. Ce promontoire constitue l'extrémité orientale d'une chaîne d'îles et d'îlots s'égrainant depuis la presqu'île de Quiberon (fig. 1). Le leucogranite à biotite et muscovite d'orientation nord-ouest/sud-est constitue le substrat commun à tous ces sites. Il est ici parsemé de filons de pegmatite mais également de micaschiste et de gneiss. Aujourd'hui recouvert de sable, ces rochers n'affleurent que localement. Le couvert végétal à l'époque néolithique ne nous est connu qu'au travers d'une analyse palynologique menée en contexte tourbeux ${ }^{2}$ à quelques mètres du gisement. Les résultats indiquent l'absence

Figure 1 - Localisation des gisements mentionnés (d'après Large, 2009)

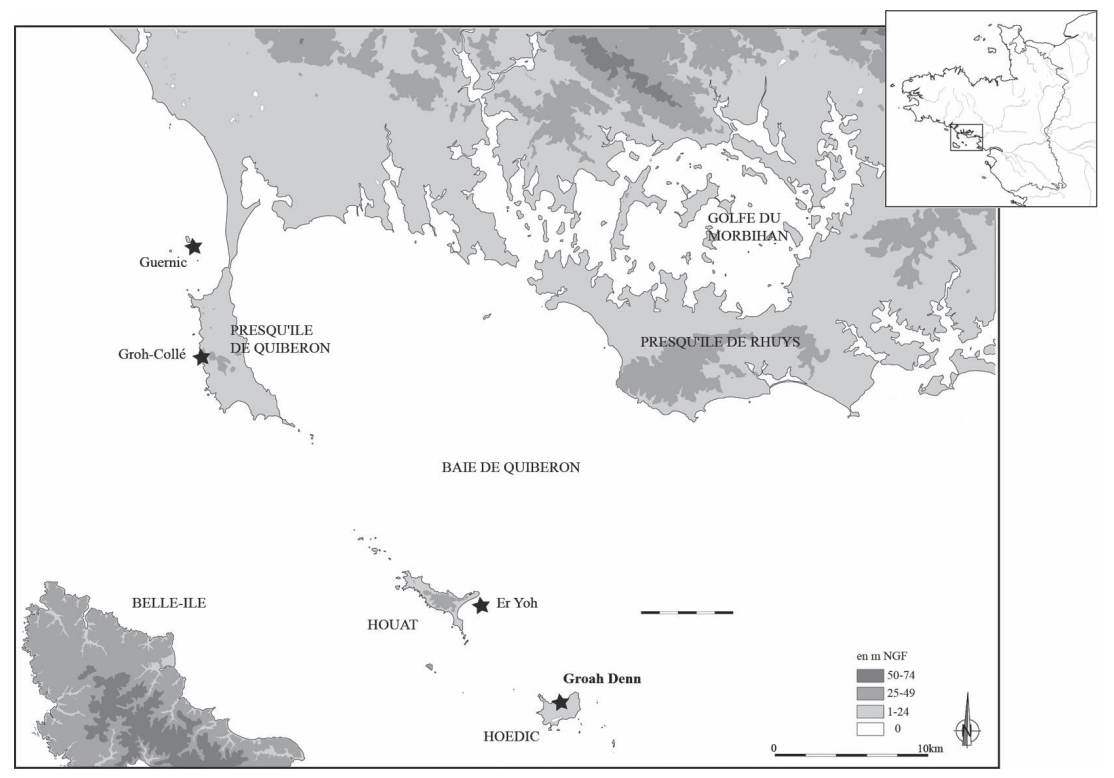

2. Delalande, Claire, Joly, Camille, VisSET, Lionel, «Évolution du paysage insulaire d'Hoedic (Morbihan) du Néolithique moyen à la période contemporaine ", Amarai, n ${ }^{\circ}$ 17, 2004, p. 17-31. 
de végétation arborée, phénomène assez classique dans un tel contexte. Le paysage néolithique est donc difficile à reconstituer. Les recherches archéologiques menées en 2009 sur le site de Groah Denn livrent néanmoins quelques informations complémentaires à ce sujet.

Le site de Groah Denn est localisé sur la pente d'Argol, au nord de l'île d'Hoëdic, à seulement quelques mètres de l'alignement mégalithique du Douet. Ce dernier fouillé de 2004 à 2006 par J.-M. Large, a soulevé de nombreuses questions concernant en premier lieu la datation mais également les groupes humains à l'origine des structures qui y ont été observées. Les mégalithes qui composent le site ont été édifiés dans la première moitié du Ve millénaire. Cependant l'organisation de la file de blocs est modifiée au cours du IV millénaire avec l'aménagement d'un mur suivant l'axe des blocs, ceci sous-tendant d'importants changements sociétaux. Afin d'étayer ces premières observations l'attention de l'équipe s'est portée sur le gisement de Groah Denn, qui présente à première vue, de grandes similarités structurelles avec le Douet; ce site se caractérise en effet par un alignement de dix blocs émergeant actuellement de la dune.

Une première campagne de sondages en 2007 concerne les deux extrémités visibles de l'alignement (fig. 2). Les résultats obtenus par la fouille de ces deux zones, au contact direct des blocs, divergent nettement. Alors que le sondage nord (Groah Denn 2) offre des blocs et un sol n'ayant pas subi de perturbations, le sondage de haut de pente (Groah Denn 1) semble montrer des structures plus récentes. Le fouilleur croit alors détecter un " rempart ${ }^{3}$ " et par là même une architecture similaire à celle du Douet. La reprise des fouilles en 2009 sur Groah Denn 1 démontre qu'il n'en est rien.

Dès lors, une importante surface $\left(117 \mathrm{~m}^{2}\right)$ a été ouverte en haut de pente, à l'extrémité sud de l'alignement, de part et d'autre de la file de blocs (fig. 2). Trois blocs mégalithiques dont deux dressés ont été mis en évidence ainsi que des séries de blocs et de galets marins venant combler l'espace entre ces pierres. Pas moins de sept couches archéologiques (dites unités stratigraphiques soit US) sont repérées en 2009 contre trois en $2007{ }^{4}$ (fig. 3). Une dune sableuse, pauvre en mobilier, recouvre l'ensemble du gisement sur 0,50 à 0,70 mètre. À sa base, un horizon sablo-limoneux, fin à grossier, brun clair (unité stratigraphique 2a) s'étend sous les blocs B4 et B1. L'étendue des quatre niveaux restants varie de deux mètres carrés (US3c) à une trentaine de mètres pour l'horizon le plus épais (US3b). Le leucogranite à biotite et muscovite qui constitue le substrat de l'île livre quelques vestiges mobiliers piégés dans ses diaclases, indiquant un rocher vraisemblablement à nu au cours du Néolithique.

Si aucune structure domestique n'est à mentionner, la présence de zones d'activités (taille : amas de débitage) et de dépôts rend ce gisement tout à

3. LARGE, Jean-Marc, Groah Denn et Lann Vihan, rapport de fouille programmée, SRA de Bretagne, Rennes, 2007, p. 20.

4. LARGE, Jean-Marc, Groah Denn, rapport de fouille programmée, SRA de Bretagne, Rennes, 2009, 47 p. 
Figure 2 - Pente d'Argol (Hoëdic, Morbihan), zones fouillées (à gauche), plan global de Groah Denn 1 (à droite)
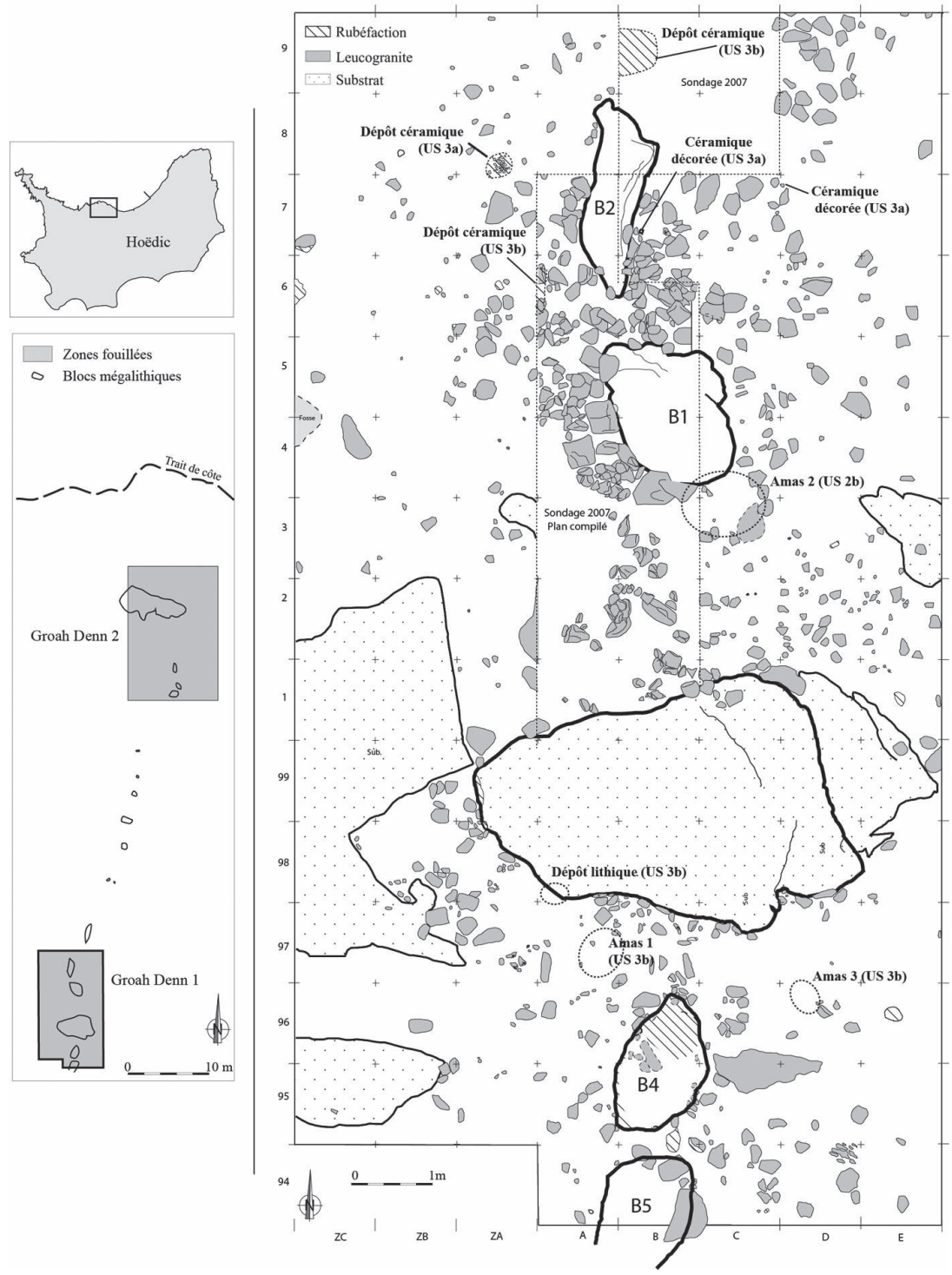

fait singulier (fig. 2). Les phénomènes de dépôts de mobilier céramique et lithique n'ont rien d'exceptionnel dans de tels contextes. En revanche, la fouille d'amas de débitage en place est, quant à elle, beaucoup plus rare. L'exploration de Groah Denn 1 a offert l'opportunité, par une fouille fine, de travailler sur l'organisation spatiale de tels ateliers. 


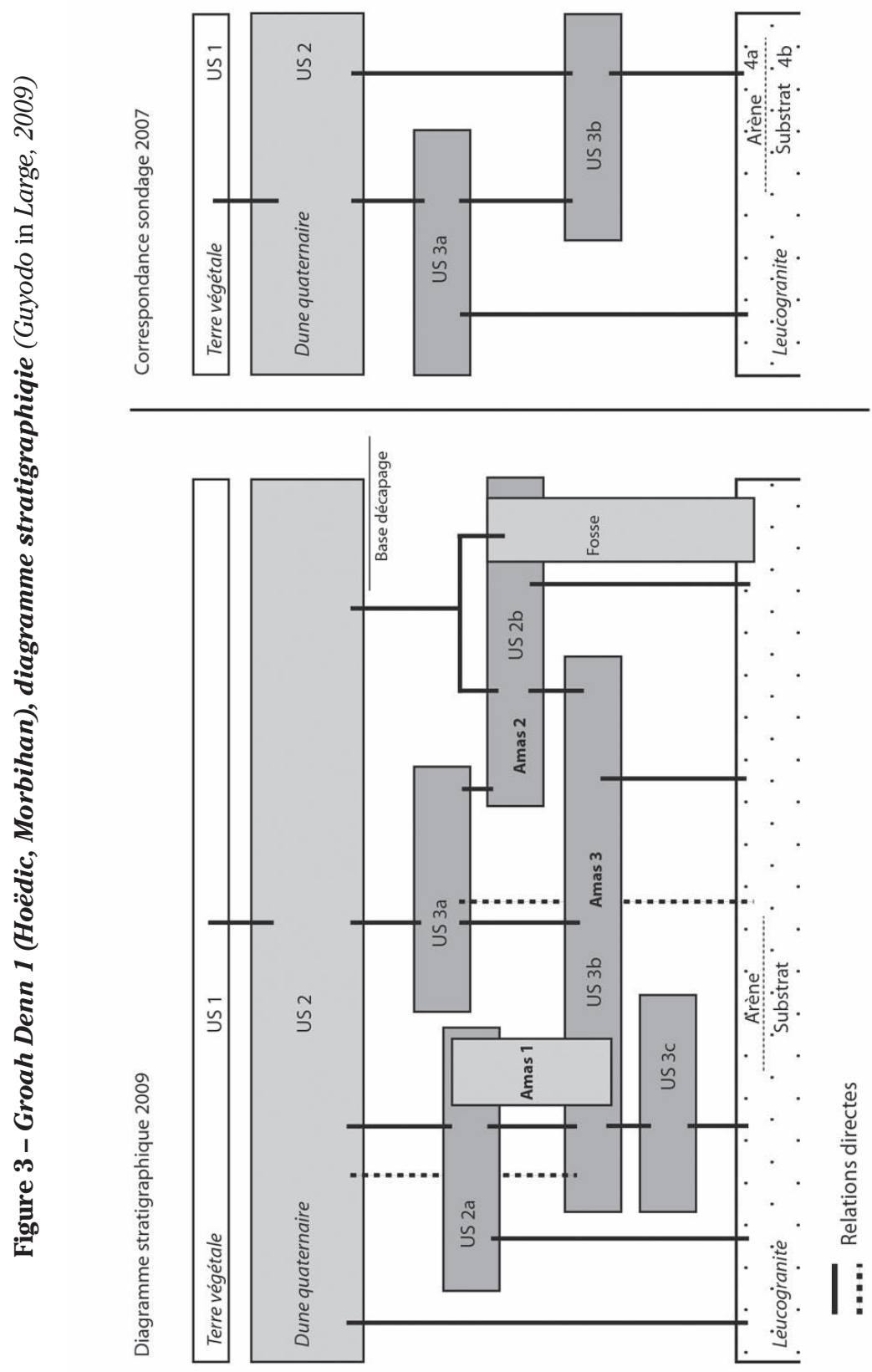




\section{La culture matérielle}

L'étude du mobilier archéologique a permis d'affiner le contexte chronoculturel de Groah Denn 1 et donc de replacer cette partie du gisement dans son contexte régional. Les résultats proposés renvoient au mobilier issu des fouilles de 2007 et 2009. Il faut cependant tenir compte des problèmes posés par le mobilier de 2007 qui, en raison d'un enregistrement en trois dimensions déconnecté de toutes couches archéologiques, n'a pu être intégré totalement à l'étude, problèmes auxquels se sont ajoutés de mauvais remontages de profils.

\section{Production céramique}

Les différentes unités stratigraphiques ont livré un corpus de 834 tessons répartis de façon disproportionnée de part et d'autre des blocs dressés. À l'est de l'alignement, les tessons sont peu nombreux et répartis sur toute la surface. À l'inverse, la partie occidentale en offre un nombre très élevé mais ils sont concentrés sur quelques mètres carrés seulement, ce qui s'explique par la fracturation en place de quelques récipients, notamment près du bloc B2 (fig. 2). Des remontages systématiques ont permis de reconstituer des profils. Ces raccords concernent des tessons de zones généralement rapprochées, suggérant non pas un rejet mais plus vraisemblablement des dépôts céramiques. Des remaniements sont toutefois à évoquer pour l'horizon supérieur (base de la dune) comme l'indiquent des raccords entre tessons provenant d'unités stratigraphiques différentes, tous découverts lors du décapage. Ce mobilier bien que très fragmenté (plus de $50 \%$ des tessons ont une surface inférieure à $2 \mathrm{~cm}^{2}$ ) a intégralement fait l'objet d'une étude typo-technologique.

La production céramique est homogène sur le gisement puisque les observations technologiques ne permettent aucune distinction ni sur le plan stratigraphique ni de nature planimétrique. Trois groupes de pâtes ont pu être distingués à la faveur d'observations à la loupe binoculaire. Ils présentent essentiellement un cortège d'inclusions minérales granitoïdes (quartz, feldspath, muscovite/quartz, muscovite/quartz, feldspath, muscovite et biotite) qui plaide en faveur d'une provenance locale de l'argile. À cet assemblage minéralogique s'ajoutent des inclusions mineures telles de la chamotte, de la coquille (pilée ou non) ou encore des végétaux. Les inclusions, de formes globalement anguleuses, ainsi que la présence de fragments de granite suggèrent l'ajout volontaire de roches broyées dans la pâte. La finesse des parois des récipients recueillis sur le site (autour de $7 \mathrm{~mm}$ ) est un fait remarquable au sein des différents niveaux de sols. Seul l'horizon $3 \mathrm{~b}$ compte un nombre plus important de tessons épais. Ces vases semblent majoritairement montés au colombin si l'on en croit les fracturations longitudinales ainsi que les cassures formant des biseaux ou des gouttières. Cependant d'autres procédés comme le modelage et le montage par plaques sont suggérés par quelques fragments. L'appréciation 
de ces différentes techniques de façonnage par observations macroscopiques n'exclut pas la pratique d'autres gestes non perçus. Le lissage, le polissage ainsi que le lustrage sont les trois principaux traitements de surface développés sur les récipients de Groah Denn 1. Le premier domine l'ensemble tandis que les deux suivants se répartissent différemment selon les couches stratigraphiques. Ainsi, les tessons polis constituent $8 \%$ du lot de l'unité stratigraphique $2 \mathrm{~b}$. Ce même niveau présente un taux de tessons lustrés important (13\%) en comparaison des niveaux 3a ou 3b (2,5 et 7 \%). Un certain soin semble porté à la surface des récipients. Les colorations constatées varient peu avec des cœurs essentiellement sombres (brun à noir), tandis que les surfaces ont des colorations variant du brun au beige en passant par le gris et l'orangé. Ces dernières remarques permettent non pas d'affirmer mais pour le moins d'évoquer une phase de cuisson en atmosphère réductrice responsable de la coloration interne sombre, et une phase de refroidissement réductrice ou oxydante; la teinte claire résultant d'une durée d'exposition à l'oxygène plus longue que la sombre.

Les quelques éléments typologiques recueillis (morphologie, décor) suggèrent la même homogénéité stylistique. Une forme complète de profil discontinu, marquée par un fond plat, au col et au bord droit à lèvre légèrement amincie, a pu être intégralement remontée (fig. $4, \mathrm{n}^{\circ} 1$ ). Ce récipient assez épais et grossier provient du dépôt reconnu à proximité du bloc dressé B2. De la même façon, les quatre profils incomplets individualisés proviennent de zones bien circonscrites. Ces dépôts ne prennent cependant pas place au sein des mêmes unités stratigraphiques (3a, 3b, 2b). Il faut donc imaginer des gestes reproduits à différents moments. La fréquentation du site par le groupe s'effectue visiblement sur un temps relativement long et en plusieurs moments distincts comme l'indique aussi la multitude des horizons stratigraphiques reconnus. Un récipient de forme globalement biconique présente quant à lui un col rentrant, un bord droit à lèvre aplanie. Il est de surcroît orné d'un motif de cannelures parallèles rectilignes alternant avec des zigzags, ornementation qui se cantonne essentiellement à la partie supérieure du vase (fig. $4, \mathrm{n}^{\circ} 2$ ). Les derniers récipients rencontrés sont beaucoup moins fournis en nombre de tessons et n'offrent donc qu'un intérêt plus limité. Il s'agit une nouvelle fois de vases à fonds plats aux bords éversés (fig. $4, \mathrm{n}^{\circ} 4$ et 5).

Indépendamment de ces ensembles, cinquante-sept tessons fournissent des indications morphologiques. Les récipients à fonds plats, débordants ou non, sont fréquents. Le déficit en fond rond s'explique quant à lui par la difficulté de les distinguer des fragments de panses et n'exclut, en aucun cas, leur présence. Les profils carénés sont également attestés par des carènes vives (fig. $4, \mathrm{n}^{\circ} 3$ ). Les bords indiquent quant à eux l'existence de cols le plus souvent concaves mais également droits (38\%) ou convexes (8 \%). Les bords sont également droits à éversés impliquant des formes relativement ouvertes pour une grande variété de lèvres (amincies, aplanies, débordantes, arrondies; fig. $5, \mathrm{n}^{\circ} 1,2,4$ à 8). L'absence d'élément de préhension et/ou de suspension est un fait marquant de cette collection. 
Figure 4 - Groah Denn 1 (Hoëdic, Morbihan), mobilier céramique (1: vase à fond plat et bord droit, 2 : vase biconique à fond plat orné de cannelures, 3 : carène, 4 et 5 : vases à fons plats et bords éversés)
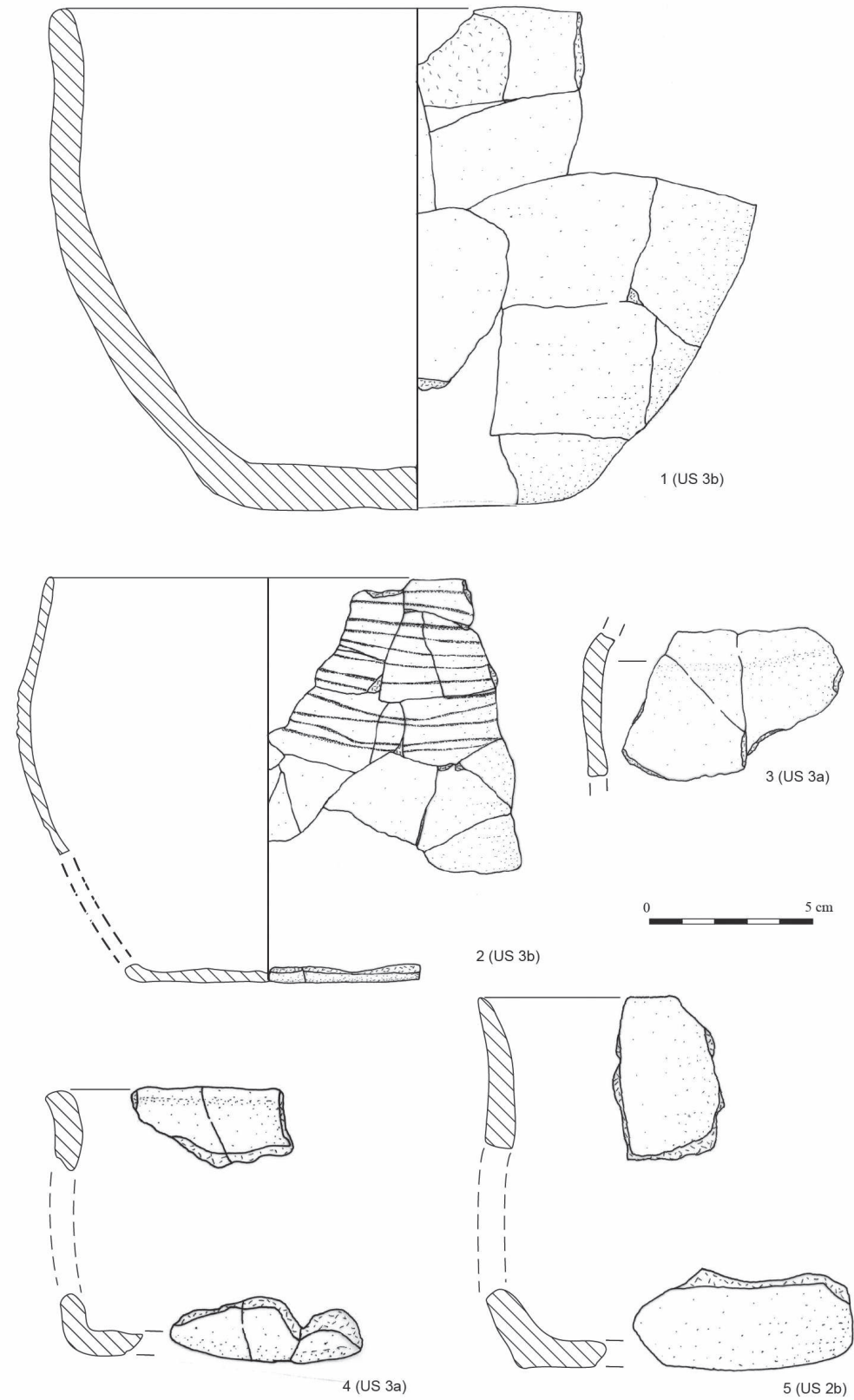
Figure 5 - Groah Denn 1 (Hoëdic, Morbihan), mobilier céramique ( 1 et 2 : bords ornés d'un cordon, 3 : fragment à motif campaniforme, 4 à 8 : bords ornés de cannelures, 9 à 12 : tessons ornés de cannelures)
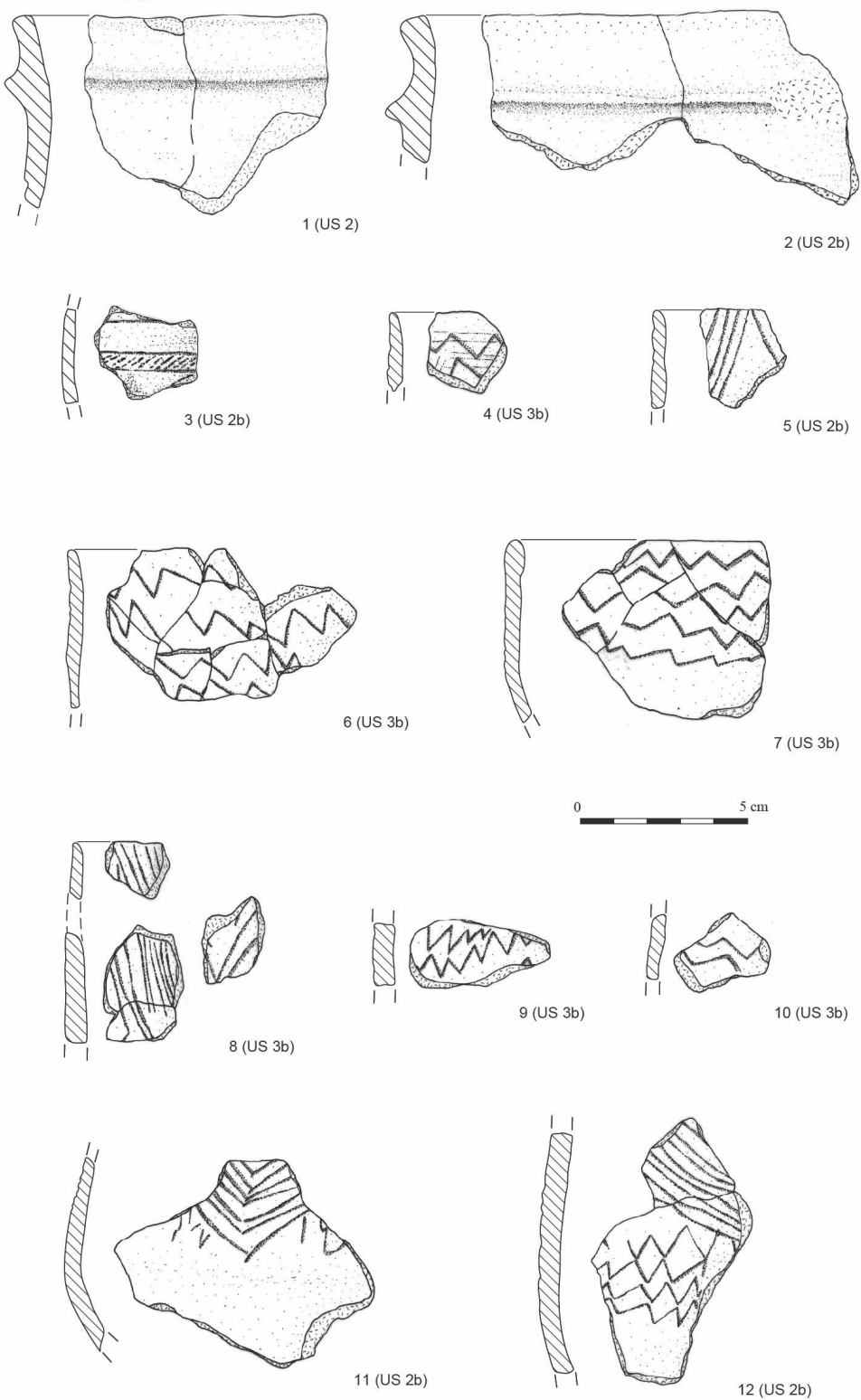
Les décors sont peu fréquents mais témoignent d'une production homogène, à une exception près. En effet, un tesson est orné de deux cannelures parallèles disposées de part et d'autre de lignes obliques parallèles imprimées à la coquille (fig. $5, \mathrm{n}^{\circ} 3$ ). Ce motif campaniforme se rencontre à la base de la dune de sable, tout comme deux fragments de bords droits à lèvres débordantes vers l'extérieur qui présentent un épais cordon préoral (fig. $5, \mathrm{n}^{\circ} 1$ et 2 ). Si l'on excepte ces éléments de périodes plus récentes, les tessons néolithiques sont plus classiquement ornés de cannelures. Localisées le plus souvent en partie haute des récipients, elles peuvent parcourir tout le col du vase ou se diviser en différents panneaux. Elles s'organisent en série de lignes parallèles droites ou encore en zigzags ou en chevrons (fig. $5, \mathrm{n}^{\circ} 4$ à 12), mêlant parfois plusieurs organisations (chevrons et lignes rectilignes).

\section{Assemblage lithique}

Le nombre de pièces lithiques recueillies sur la zone fouillée est important (19160 pièces) et s'explique en partie par l'activité de taille du silex sur le gisement (fig. 2). Trois amas de débitage se distinguent nettement dans cette collection et ils seront traités individuellement. Le premier, au sud de l'affleurement leucogranitique, a fait l'objet d'une fouille fine. Il présente la particularité d'être complété à l'ouest par un dépôt lithique spécifique (lames de hache polie et galet biseauté). L'amas $\mathrm{n}^{\circ} 3$ se trouve également au sud de l'affleurement et se raccorde au même horizon stratigraphique (3b). Le dernier amas ( $\left.\mathrm{n}^{\circ} 2\right)$, localisé contre le bloc B1, a livré la plus grande concentration de mobilier lithique et s'inscrit dans un niveau plus récent que les précédents (US 2b; fig. 2). Au sud de l'affleurement, outre les amas de débitage, la densité de pièces se fait plus importante que sur l'ensemble de la zone explorée.

Les matières premières employées s'avèrent principalement locales. À $81 \%$, c'est le silex qui domine la série, toutes unités stratigraphiques confondues. Il prend essentiellement la forme de galets côtiers provenant des anciennes plages perchées de galets de la presqu'île quiberonnaise (îlot de Téviec, etc.). Quelques pièces en silex importés ou encore en chaille viennent ponctuellement compléter l'ensemble. L'approvisionnement en quartz, très prisé pour le macro-outillage, est également facilité par la présence de filons parcourant l'île d'Hoëdic. De la même façon, la pegmatite est accessible à quelques mètres seulement de la zone fouillée, puisqu'elle affleure en contrebas. L'exploitation de ces roches n'a donc rien d'étonnant. Le substrat local leucogranitique est également utilisé pour le macrooutillage, le plus souvent pour les outils de mouture. Outre ces roches locales, des matériaux, moins fréquents, de provenance plus lointaine, sont attestés tels le schiste, les quartzites, la phtanite, le grès ou encore la dolérite. Cette dernière provient très certainement d'un réseau d'échange propre à la circulation de produits finis que sont les lames de hache polie. 
Le débitage, quel que soit le lot étudié, est assez uniforme. Les nucléus indiquent une production très majoritairement tournée vers l'extraction d'éclats. L'obtention de supports laminaires (lames, lamelles), beaucoup plus anecdotique, relève de la même chaîne opératoire. Le débitage est le plus souvent bipolaire ou unipolaire et s'effectue par percussion posée sur enclume ${ }^{5}$ et, dans une moindre mesure, par percussion directe au percuteur dur ${ }^{6}$. À l'exception d'un fragment de lame vraie, ces supports sont pour l'essentiel des éclats à tendance laminaire $(0,2 \%$ de production laminaire). La réalisation d'éclats découle d'une chaîne opératoire simple et rapide à mettre en œuvre. Ainsi que l'indiquaient au préalable les nucléus, la percussion posée sur enclume est omniprésente tandis que la percussion directe dure n'intervient que ponctuellement. Les talons, lorsqu'ils sont présents, témoignent de plans de frappe pas ou peu préparés. Le débitage depuis un plan de frappe voire deux est le plus répandu. Des accidents de taille interviennent aux différents stades de la chaîne opératoire, témoignant de maladresses du tailleur et/ou de l'emploi d'une matière première de moindre qualité. Les esquilles sont présentes en quantité variable au sein des différents lots.

Tous les types de supports sont concernés par la retouche et les traces d'usure (micro-esquillements). Si l'on excepte les unités stratigraphiques 2 et 3c qui ont fait l'objet d'un ramassage lacunaire, le taux de pièces retouchées (hors outils) est identique au sein des différents niveaux ( 0,8 à $1,7 \%$ des lots). De la même façon, le taux de supports utilisés bruts oscille entre 1,6 et $1,7 \%$ pour chacun des lots. Seul l'horizon 3a, en dépit de son total de 422 pièces, atteint $4 \%$ de supports micro-esquillés. Cette disproportion subsiste dans le taux d'outillage puisqu'il s'élève à 4,3\% contre 1,5 à $2 \%$ au sein des autres niveaux.

La part des outils est quantitativement faible (1,5 à 4,3 \% des différents lots). Les pièces esquillées ${ }^{7}$ dominent assez largement l'outillage. Ces outils, de forme généralement quadrangulaire, se caractérisent par de petits enlèvements bifaciaux - esquillements d'utilisation et/ou de percussion - le plus souvent sur deux extrémités opposées, plus ponctuellement sur une, trois ou quatre. La réutilisation des nucléus est courante pour la confection de celles-ci (fig. $6, \mathrm{n}^{\circ} 1$ à 6 ). Il s'agit bien d'un réemploi de ces nucléus et non de simples stigmates laissés par l'usage de la percussion posée sur enclume ${ }^{8}$ car les esquillements sont ici trop importants pour résulter du simple débitage. Les traces d'usure ne correspondent pas tou-

5. GuYodo, Jean-Noël, MARCHAND, Grégor, " La percussion bipolaire sur enclume dans l'Ouest de la France de la fin du Paléolithique au Chalcolithique : une lecture économique et sociale ", Bulletin de la Société Préhistorique Française, t. 102, n 3, 2005, p. 539-549.

6. TIXIER, Jacques et al., Préhistoire de la pierre taillée, T.1 : terminologie et technologie. Paris, éd. du Cercle de Recherches et d'Etudes Préhistoriques, 1980, 120 p.

7. LE BRUN-RICALENS, Foni, "Les pièces esquillées : état des connaissances après un siècle de reconnaissance ", Paléo, 18, 2006, p. 95-114.

8. GuYodo, Jean-Noël, MARChAND, Gregor, " La percussion bipolaire sur enclume... ", op. cit. 
Figure 6 - Groah Denn 1 (Hoëdic, Morbihan), mobilier lithique

(1 à 6 : pièces esquillées sur nucleus, 7 à 9 : grattoirs, 10 à 12 : perçoirs, 13 à 14 : armatures microlithiques, 15 : armature tranchante Sublaines)
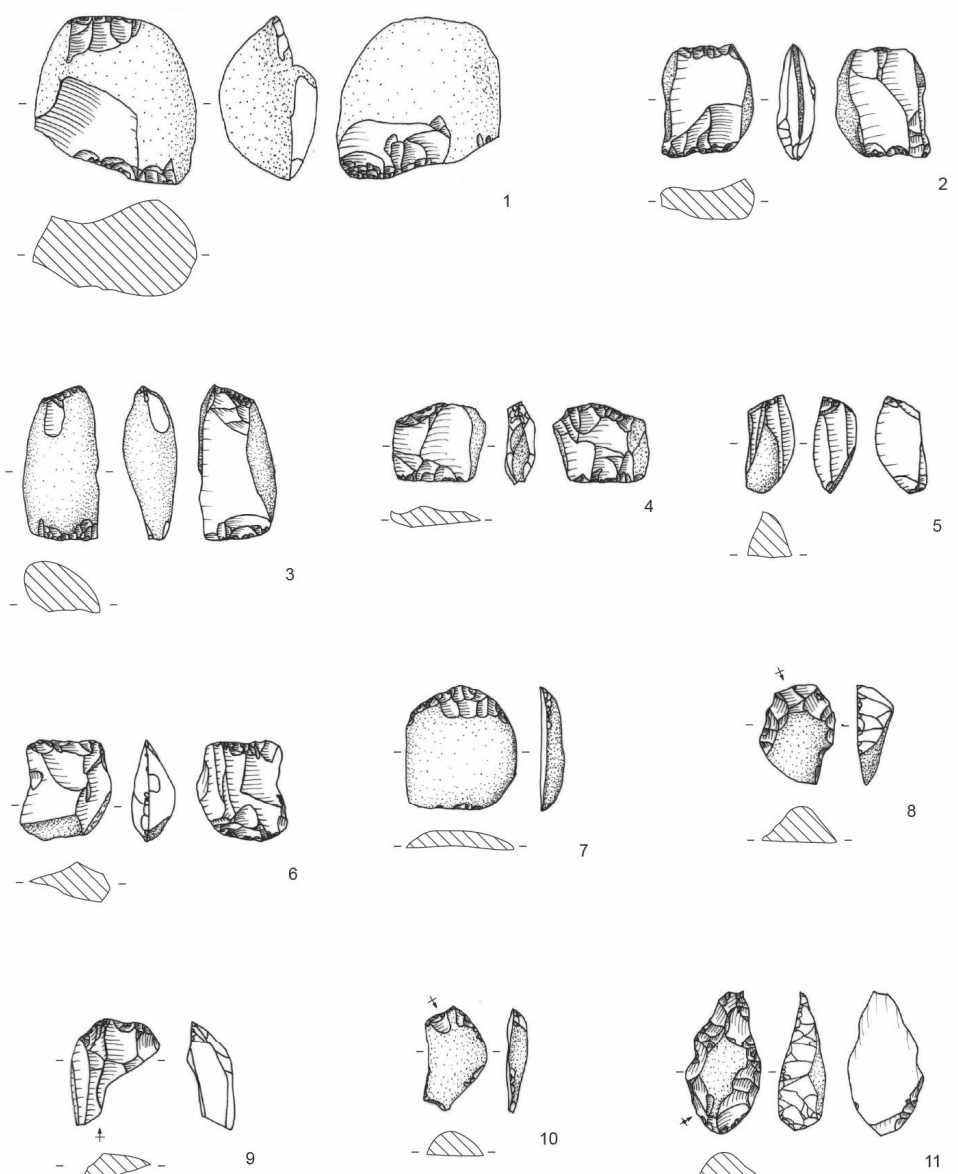

9
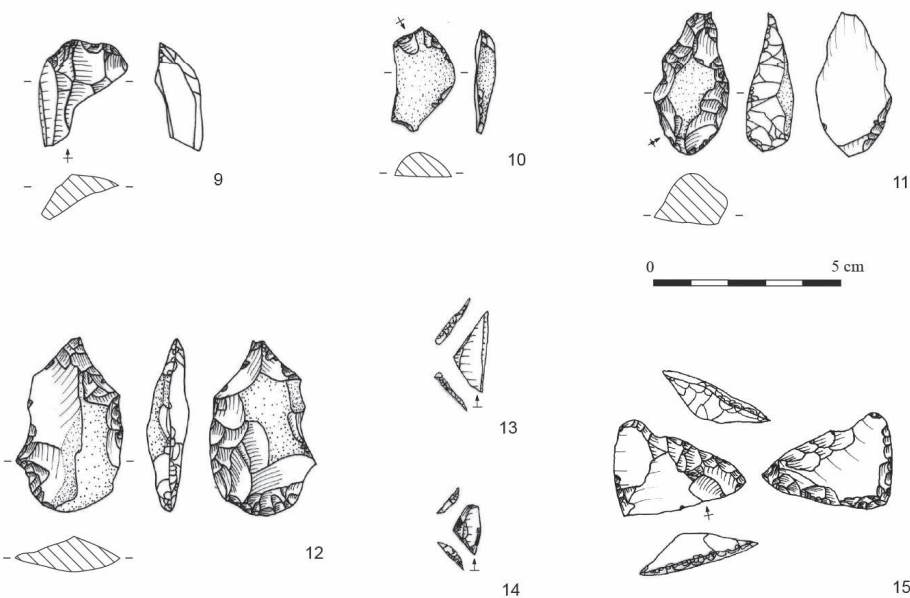
jours à l'orientation des enlèvements. La présence de cortex sur les bords facilite la prise à pleine main de ces pièces dont l'utilisation s'effectue sur deux zones actives opposées. Ces outils, dont l'existence est attestée dès le Paléolithique, soulèvent de nombreuses questions quant à leur fonction. Ils sont ainsi potentiellement liés au travail du bois, de l'os, de l'ivoire, de coquille, mais une utilisation en pièce intermédiaire dans le débitage n'est pas exclue. Leur présence suppose la tenue d'une activité particulière sur le gisement qui, en l'absence d'études tracéologiques plus poussées, est encore impossible à déterminer. Les grattoirs, bien que moins fréquents, constituent une part non négligeable de l'outillage (12\%; fig. 6, nº 7 à 9). Ils sont obtenus par retouches directes abruptes continues de l'extrémité de tous types de supports. La morphologie de ces derniers est relativement trapue bien que leurs dimensions varient quelque peu ( $29 \times 23 \times 10 \mathrm{~mm})$. Les supports des perçoirs sont aussi diversifiés mais de formes allongées ( 31 x 20 x 9 mm; fig. $6, \mathrm{n}^{\circ} 10$ à 12). La partie active de ces outils est façonnée par retouches directes abruptes auxquelles s'adjoignent parfois des retouches inverses. Les coches retouchées s'approchent sur le plan morphométrique des perçoirs ( $31 \times 23 \times 7 \mathrm{~mm}$ ). Les denticulés, les racloirs ainsi que les troncatures sur lames paraissent quant à eux secondaires dans le corpus d'outils. Les armatures de flèches, bien que rares, constituent des marqueurs chronologiques des plus intéressants. Deux d'entres elles sont en réalité des triangles, dont un isocèle, relevant du Mésolithique ancien (fig. $6, n^{\circ} 13$ et 14). Leur position à la base de la dune, de même que le caractère patiné de l'une d'entre elle, indiquent indubitablement une position secondaire. Une armature de flèche tranchante (US 3b) se rapproche du type dit Sublaines ${ }^{9}$ (fig. $6, \mathrm{n}^{\circ} 15$ ), par ses retouches inverses semi-abruptes à abruptes et les retouches écailleuses amincissant la face supérieure de la pièce. Façonnée sur éclat de galet côtier de silex, cette pièce témoigne de contact fort avec les groupes largement plus continentaux du Néolithique récent.

L'activité de taille in situ est bien attestée par le macro-outillage. Les percuteurs (galets de quartz) et les enclumes attestent du débitage sur le gisement et font le lien avec les données techniques évoquées. Des outils composites impliquent également une gestion tout à fait particulière de ces pièces qui sont d'usages multiples : des enclumes présentent ainsi des plages de percussion, relatives à un usage en tant que percuteur (fig. 7, $\mathrm{n}^{\circ} 3$ ), ou encore des plages polies liées à une activité de mouture (fig. 7, $\mathrm{n}^{\circ} 2$ ). L'extraction de matériaux lithiques volumineux (mégalithes du site?) peut également être évoquée avec la présence d'un coin en pegmatite. Les macro-outils liés aux activités de mouture témoignent d'activités d'ordre domestique : les meules (fragments souvent de faibles dimensions) et les molettes, toutes en leucogranite, sont fréquentes. Un poids de pêche en pierre vient renforcer l'hypothèse d'activités de subsistance halieutique en

9. Noutl A. et al., "L'ossuaire néolithique d'Eteauville commune de Lutz en dunois (Eure-et-Loir) ", Bulletin de la Société préhistorique française, t. 62, 1965, p. 576-648. 
Figure 7 - Groah Denn 1 (Hoëdic, Morbihan), mobilier lithique (1: galet biseauté, $2:$ meule/percuteur, $3:$ enclume/percuteur, 4 : poids de pêche, 5 et 6 : lames de haches polies)
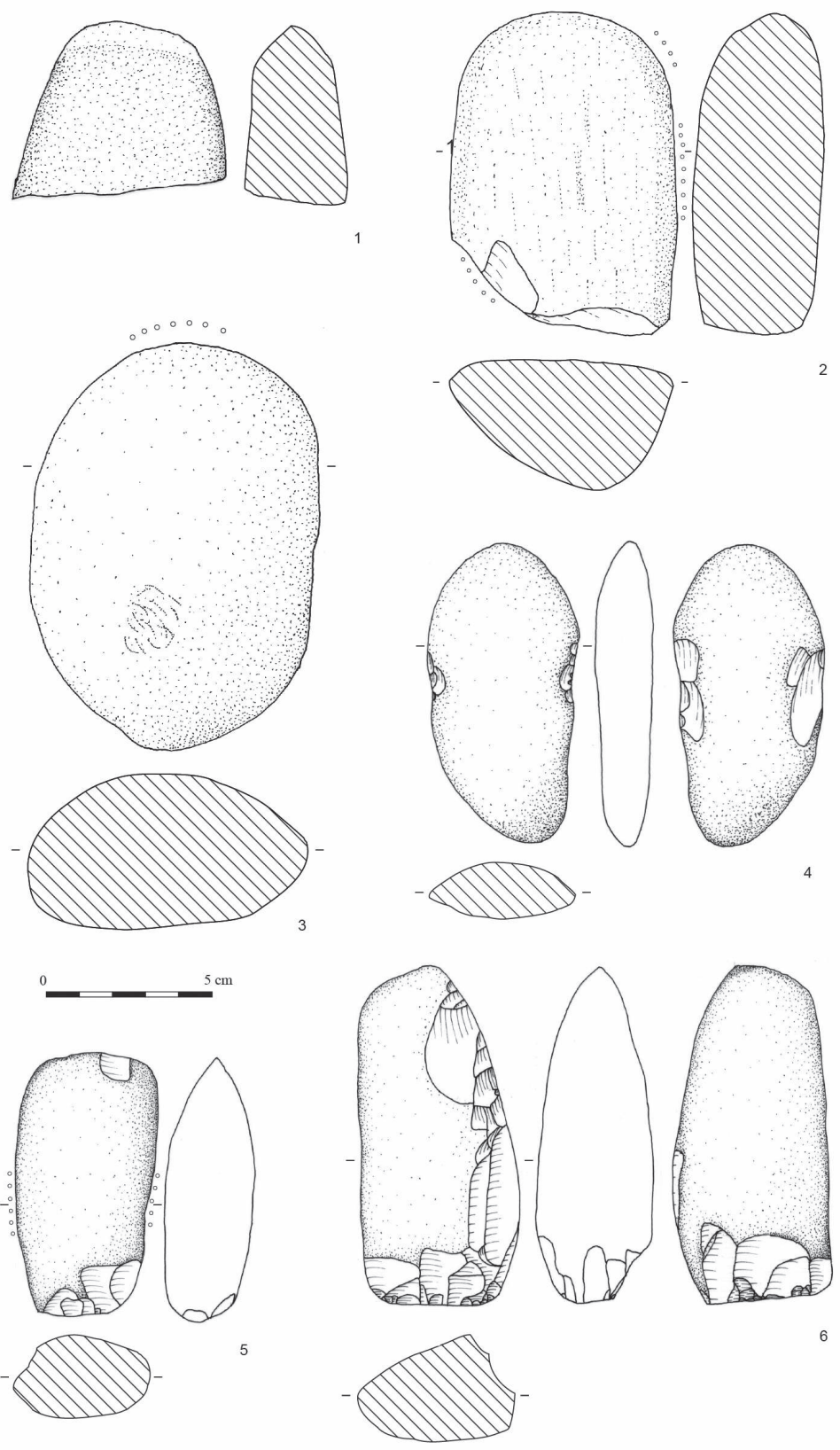
lien avec l'occupation (fig. 7, $\mathrm{n}^{\circ}$ 4). Des éléments tels que les galets biseautés (fig. $7, \mathrm{n}^{\circ} 1$ ) ou encore des blocs présentant des polis d'usure sur une ou plusieurs extrémités ou face(s) ont une vocation dans le cas présent non définissable, peut-être en lien avec le débitage pour les premiers ou la confection de récipients céramiques pour les seconds.

Indépendamment de cet ensemble relativement classique, le dépôt lithique mentionné au sud de l'affleurement (carré A98) comporte trois macro-outils : une lame de hache polie en roche métamorphique, un fragment de lame de hache polie en dolérite (fig. $7, n^{\circ} 5$ et 6 ) et un galet biseauté en leucogranite. Ces pièces permettent de replacer Groah Denn 1 dans un plus large réseau d'échange puisque les deux lames de haches polies ne relèvent pas d'une matière première identifiée sur Hoëdic mais d'importation de produits finis continentaux.

\section{Les amas de débitage}

Une fouille d'amas de débitage, de surcroît en contexte insulaire, est un fait assez exceptionnel. Afin de permettre une analyse pertinente, une fouille fine a été menée sur les amas $\mathrm{n}^{\circ} 1$ et 2 . Des raccords physiques ont également été tentés sur les différentes pièces recueillies afin de déterminer les types de supports recherchés. Aucun tamisage n'a été effectué sur le site. Le protocole développé avait pour but d'envisager les modalités et la durée d'une telle activité sur un site déconnecté de toutes zones d'habitations.

Le premier amas de débitage mis en évidence (US 3b) ne compte que 360 pièces. Vingt nucléus ont tout de même pu être isolés, essentiellement dévolus à l'obtention d'éclats. Ainsi que le suggèrent les blocs de matière première, la production laminaire est inexistante dans cet amas. Tous les types d'éclats sont représentés avec une nette prédominance des éclats de début de débitage. mais d'autres éclats prélevés se rattachent au terme de la séquence gestuelle. Ils sont majoritairement extraits par percussion posée sur enclume mais également par percussion directe dure depuis un ou deux plans de frappe qui ne sont pas ou peu préparés. Les esquilles sont ici en quantité bien plus importante que sur l'ensemble du gisement puisqu'elles représentent 17 \% des pièces en silex, ce qui n'a rien d'étonnant au regard de l'activité pratiquée. Les pièces retouchées et les pièces micro-esquillées sont au nombre de quatre. Un grattoir, seul outil, se distingue de cet ensemble correspondant à des déchets de taille.

L'amas $n^{\circ} 2$, au sud du bloc B1 (US 2b), est lui plus fourni avec 2598 pièces. Seuls deux des 199 nucléus découverts montrent des négatifs de produits laminaires en plus des éclats traditionnellement recherchés. La percussion posée sur enclume y est une nouvelle fois la technique de débitage prédominante, suivie de la percussion directe dure. Comme dans l'amas $n^{\circ} 1$, les éclats de début de débitage sont les plus fréquents. Le taux laminaire s'élève ici à un niveau ridiculement faible de 0,2 \%. La chaîne 
opératoire engagée est une fois encore courte et simple. Des accidents, rares, interviennent à différents moments de la séquence gestuelle. Les esquilles ne représentent ici que $3 \%$ du lot, taux bien faible en comparaison de l'activité développée mais tout de même nettement plus important que sur l'ensemble du gisement. Douze supports sont retouchés et vingt-six micro-esquillés. L'outillage est bien peu représenté avec seulement deux pièces esquillées.

La répartition spatiale du mobilier lithique au sein de l'amas de débitage témoigne d'une organisation particulière de cet espace de production. Ainsi, le ramassage par maille fine $\left(20 \mathrm{~cm}^{2}\right)$ révèle l'existence de zone de concentration et à l'inverse de zone vide de mobilier (fig. 8). L'extrémité nord-est de l'amas $\mathrm{n}^{\circ} 2$ présente ainsi un espace dépourvu de mobilier, qui pourrait correspondre à l'emplacement du tailleur. Au contraire, la zone sud/sud-ouest, présente une forte densité de pièces lithiques résultant de la projection des pièces lors du débitage. Les macro-outils sont quant à eux disposés non dans l'amas de pièces lui-même mais à proximité. Les raccords physiques, nombreux, mettent en exergue la forte proportion de supports abandonnés au cours de la séquence gestuelle.

Ces zones bien circonscrites sont donc dédiées à la seule activité de taille, aucun élément céramique n'ayant en effet été observé au sein de ces amas. La quasi-absence de sédiment séparant le grand nombre de pièces lithiques recueillies laisse envisager une constitution de ces amas dans un laps de temps relativement court (de l'ordre de la journée pour un individu). La chaîne opératoire développée reste courte et simple, dévolue à la production d'éclats. Le mobilier propre à ces zones d'activités ne diffère donc en rien des productions observées sur l'ensemble du gisement. La faible importance de l'outillage s'explique assez logiquement puisque les pièces ici produites sont vouées à être utilisées dans d'autres contextes tel l'habitat. Ces amas de débitage relèvent ainsi de la seule production; les déchets et rares macro-outils liés à la confection des différents outils et/ou supports sont ainsi les seuls éléments disponibles. La retouche des supports semble assez intense dans l'amas 1 si l'on en juge par la forte quantité d'esquilles. L'amas 2 s'en distingue quelque peu puisque ces dernières y sont plus faiblement représentées : la fabrication des outils pourraient avoir eu lieu ailleurs, ce qui distingue ces deux amas en termes de gestion des supports et de production des outils. On peut donc supposer qu'au sein de ces deux zones, les gestes effectués et les finalités recherchées divergent, sans toutefois se différencier de façon stricte. Dans de tels contextes, aborder le type de produit confectionné est un exercice hasardeux auquel nous ne nous risquerons pas ici. Les rares outils rencontrés au sein de ces amas relèvent de différents types (pièces esquillées, grattoirs) et ne permettent en aucun cas d'évoquer la production d'une catégorie de pièces en particulier. 
Figure 8 - Groah Denn 1 (Hoëdic, Morbihan), organisation spatiale des hamas de débitage, $n^{\circ} 2$ (en haut) et $n^{\circ} 1$ (en bas)

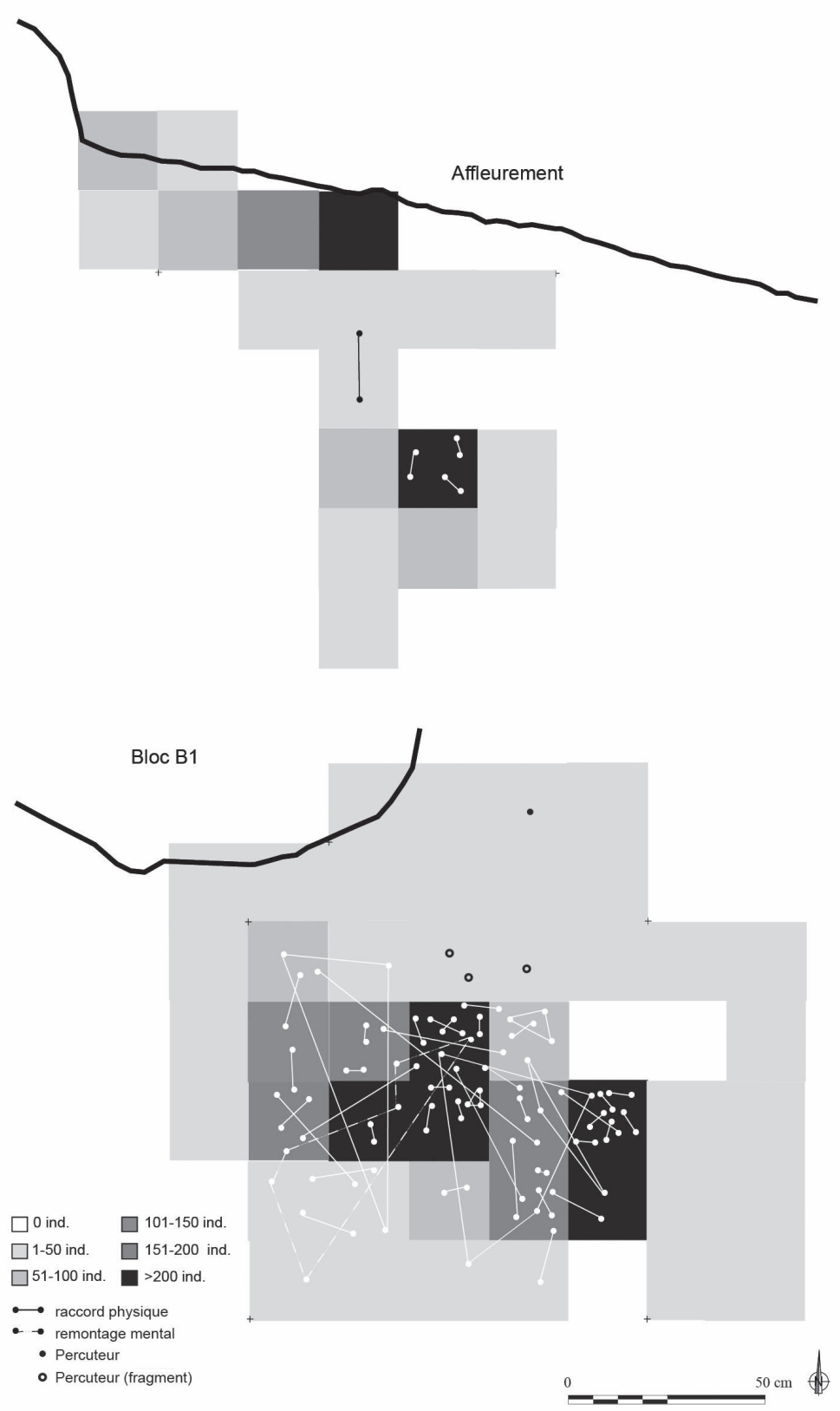




\section{Un gisement insulaire du Néolithique récent}

\section{Apport sur le Néolithique récent régional}

L'ensemble lithique et céramique recueilli renvoie, tant sur le plan technologique que typologique, au Néolithique récent régional (3800-2900 avant J.-C.). Le site de Groah Denn 1 se situe au cœur de la zone nucléaire du Groh Collé, groupe qui s'étend sur le littoral morbihannais et tout particulièrement autour de la presqu'île quiberonnaise ${ }^{10}$. Quelques rares éléments, en position secondaire, témoignent néanmoins d'occupations, ou pour le moins de passages, antérieurs mais également postérieurs. La base de la dune livre ainsi deux pièces lithiques du Mésolithique ancien (triangles) et un petit lot de tessons campaniformes. Un fragment orné à la coquille d'un motif bien connu ne pose pas de problèmes d'attribution ${ }^{11}$. Deux bords ornés d'un cordon, pouvant aisément se raccorder à une grande urne, sont plus ubiquistes et pourraient, à défaut de renvoyer au Campaniforme, se rattacher à des productions de l'Age du Bronze ancien ou moyen ${ }^{12}$.

Ces indices matériels permettent non seulement d'élargir le spectre chronologique des fréquentations du gisement mais encore d'évoquer brièvement la mise en place du couvert sédimentaire. L'absence d'éléments postérieurs à l'Âge du Bronze plaide en faveur d'une installation ancienne de la dune de sable sur le gisement, ce que corroborent les affirmations proposées par les géographes et géomorphologues ${ }^{13}$ et qui a été déjà souligné pour d'autres gisements contemporains du littoral sud-morbihannais ${ }^{14}$. Les analyses paléo-environnementales mentionnées en introduction trouvent ici un écho des plus intéressants. Le sable n'a recouvert l'île d'Hoëdic ainsi que le littoral sud-armoricain qu'au $\mathrm{II}^{\mathrm{e}}$ millénaire avant J.-C., sans doute lors de la régression/transgression marine fin Bronze ancien/début Bronze moyen, ce qui laisse imaginer un couvert végétal bien différent de l'actuel. Ce biais stratigraphique qui parait relativement simple pour la datation de certains gisements avait déjà été suggéré au terme de la fouille partielle du dolmen de Port-Blanc puis de la nécropole de Thinic à Saint-PierreQuiberon ${ }^{15}$ mais avait semble-t-il été quelque peu négligé sur le moment puisque le sujet faisait polémique, puis oublié par la suite par les archéologues pendant plusieurs décennies.

10. GuYodo, Jean-Noël, Les Assemblages lithiques des groupes néolithiques sur le Massif armoricain et ses marges, thèse de doctorat multigraphiée, Université de Rennes I Beaulieu, 2001, 466 p.

11. SALANOVA, Laure, La Question du campaniforme en France et dans les iles anglo-normandes, productions, chronologie et rôle d'un standard céramique, Paris, CTHS/SPF, 2000, 391 p.

12. Billard, Cyrille, Blanchet, Jean-Claude, Talon, Marc, "Origine et composante de l'Age du Bronze ancien dans le Nord-Ouest de la France ", dans : MoRDANT Claude et GAIFFE Olivier (dir.), Cultures et sociétés du Bronze ancien en Europe, Paris, CTHS, 1992, p. 579-602.

13. Roul, Thierry, Les dépôts littoraux quaternaires fossiles de la presqu'île de Quiberon, mémoire de maîtrise multigraphié, IGARUN, Université de Nantes, 1991, 171 p.

14. GaIllard, Felix, "Rapport sur les fouilles du cimetière celtique de l'île de Thinic (15 août 1883) ", Bulletin de la Société polymathique du Morbihan, 27, 1883, p. 231-240. 
Les motifs décoratifs rencontrés sur les céramiques sont typiques du Néolithique récent et plus particulièrement du Groh Collé tel qu'il a pu être défini par G. Bailloud ${ }^{15}$. Les cannelures parallèles prennent place essentiellement en partie haute des récipients et consistent en des lignes droites à ondulées, mêlant parfois plusieurs organisations disposées en panneaux. Les rares formes complètes observées sont plus ubiquistes. Ainsi la forme biconique mise en évidence ici n'est pas sans rappeler un vase du niveau supérieur du dolmen de Conguel à Quiberon ${ }^{16}$. Le même type de récipient est néanmoins attesté sur le site éponyme de Groh Collé ainsi qu'à PortBlanc (Saint-Pierre-Quiberon, Morbihan) ou encore à Butten-er-Hah ${ }^{17}$ (Groix, Morbihan). La proximité stylistique du Groh Collé et du Conguel, admise de longue date, pose ici problème et rend difficile une attribution stricte de Groah Denn à l'un ou l'autre.

L'assemblage lithique est lui beaucoup plus singulier et permet véritablement de trancher en faveur du groupe de Groh Collé puisqu'il en propose toutes les caractéristiques ${ }^{18}$. La forte proportion de matière première locale engagée aussi bien pour l'obtention de supports que de macro-outils est une donnée déjà évoquée pour d'autres sites du groupe tels Er Yoh ${ }^{19}$ (Houat, Morbihan). Groh Collé ${ }^{20}$ ou encore Guernic ${ }^{21}$ (SaintPierre-Quiberon, Morbihan). L'exploitation de galets côtiers de silex y est prépondérante. Cette matière première est disponible en quantité non négligeable à proximité de tous ces gisements littoraux. Les importations plus lointaines de matières comme de produits finis restent, elles, très exceptionnelles. La chaîne opératoire développée sur le site de Groah Denn 1 ne diffère en rien de celle observée sur d'autres sites du groupe : elle s'avère simple et courte, privilégiant la technique de la percussion posée sur enclume. Les éclats sont les supports principalement recherchés au détriment des produits laminaires. Ces derniers supports ne font par ailleurs en aucun cas l'objet d'une production spécialisée; leur obtention relevant plus de l'opportunisme lors du débitage d'éclats. Outre ces critères techniques, le corpus d'outil est également relativement classique pour la période : la

15. Bailloud, Gérard, "Les céramiques cannelées du Néolithique armoricain ", Bulletin de la Société Préhistorique Française, t. 72, 1975, p. 343-367.

16. L'HelgouAc'H Jean, "Le dolmen de Conguel en Quiberon (Morbihan) ", Bulletin de la Société Préhistorique Française, t. 59, fasc. 5-6. Paris, 1962, p. 371-381.

17. PollÈs Renan, "Le style de Conguel : nouveaux éléments ", Études et Travaux, Bulletin de la Société Préhistorique Française, vol. 83, n 11-12, 1986, p. 452-469.

18. GuYodo, Jean-Noël, Les Assemblages lithiques..., op. cit.

19. GuYodo, Jean-Noël, "Installations néolithiques et gauloises à Er Yoh (Houat, Morbihan) : état de la question ", Collectif, Marthe et Saint-Just Péquart, archéologues des îles, de Houat à Hoëdic, 1923-1934, Melvan, la revue des deux îles, n 4, 2007, p. 229-250.

20. Guyodo, Jean-Noël, L'habitat néolithique de Groh-Collé à Saint-Pierre-Quiberon (Morbihan), Document Final de Synthèse de fouille programmée, SRA de Bretagne. Rennes, 2008.

21. GuYodo, Jean-Noël, "L'atelier de débitage de Guernic (Saint-Pierre-Quiberon, Morbihan) : résultats des campagnes 1998-1999 ", Bulletin de l'AMARAI, n 13, 2000, p. $43-64$. 
forte proportion de pièces esquillées est un fait déjà mentionné sur le site éponyme. Les nucléus sont les supports préférentiellement engagés pour ce type de pièces. Les grattoirs, perçoirs et coches retouchées s'ajoutent à cet ensemble traditionnel. Seule l'armature de flèche de type dit Sublaines dénote au sein de la série lithique puisque ce type de pointes de projectile est plus fréquent dans le Bassin moyen de la Loire. Son façonnage sur matière première locale (galet côtier de silex) relève plus vraisemblablement d'une imitation (transfert) que d'une importation. Il est toutefois intéressant d'observer sa présence désormais bien loin de leur zone de répartition classique. Les armatures Sublaines sont également attestées sur des sites relevant du Groh Collé et plus particulièrement sur les enceintes fossoyées du sud de la Loire, les Prises à Machecoul ${ }^{22}$ (Loire-Atlantique) et les Gatineaux à Saint-Michel-Chef-Chef ${ }^{23}$ (Loire-Atlantique). Un perçoir typologiquement Moulin-de-Vent caractéristique du Peu-Richard ${ }^{24}$, sur galet côtier de silex, à Er Yoh ${ }^{25}$ (Houat, Morbihan) avait déjà suggéré l'existence de liens entre groupes du Massif armoricain et ceux du Centre-Ouest de la France.

L'assemblage lithique recueilli sur le site de Groah Denn 1 renvoie sans conteste au groupe néolithique récent régional de Groh Collé. La production céramique met en exergue la proximité des deux styles Conguel et Groh Collé qui sont ici indiscernables l'un de l'autre. Ces derniers offrent des caractéristiques typo-technologiques similaires au sein des mêmes unités stratigraphiques. Séparer ces deux styles paraît donc bien difficile et peu pertinent.

\section{Statut du gisement}

L'absence de structures relatives à l'habitat à proximité de l'alignement mégalithique pose d'ores et déjà la question du statut de ce gisement. Les différentes prospections n'ont en effet révélé qu'un corpus important de mégalithes pour peu d'éléments pouvant se raccorder à de véritables sites domestiques. La mise en évidence de zones de dépôt tant céramiques que lithiques rend d'autant plus complexe sa compréhension. La présence d'un habitat peu éloigné du site est un fait que l'on ne peut totalement exclure au regard du mobilier. Des activités domestiques se manifestent par le biais des outils de mouture ou encore de pêche.

22. Goudissard, Simon, Les assemblages lithiques du sud du Pays de Retz au Néolithique, l'exemple du site des Prises à Machecoul (Loire-Atlantique), mémoire de Master 1, Université de Rennes 2, 2008, 95 p.

23. GuYodo, Jean-Noël, Les Assemblages lithiques..., op. cit.

24. FOUÉRÉ, Pierrick, Les Industries en silex entre néolithique moyen et campaniforme dans le nord du Bassin aquitain. Approche méthodologique, implications culturelles de l'économie des matières premières et du débitage, thèse de doctorat, Université de Bordeaux-I, 1994, 2 vol.

25. GuYodo, Jean-Noël, "Installations néolithiques et gauloises... ", op. cit. 
Les dépôts céramiques et lithiques sont un trait tout à fait particulier mais pas inconnu sur l'île d'Hoëdic pour la période néolithique. Ainsi l'alignement du Douet, distant de quelques dizaines de mètres, livre le même type d'informations pour un contexte plus ancien, Néolithique moyen I ${ }^{26}$. Ces dépôts prennent place en des zones bien spécifiques du gisement, à proximité directe des blocs verticalisés et de l'affleurement massif. Ils disparaissent totalement pour peu que l'on s'éloigne de l'alignement. Il s'agit donc de gestes volontaires, loin d'être anodins : l'hypothèse de dépôts de fondations ou encore de dépôts votifs peut être avancée, sans certitude toutefois.

Les amas de débitage font état d'une production en tout point semblable, tant sur le plan technique que typologique, à celle qui a été mise en évidence sur l'ensemble du site. Tous les éléments liés à la taille sont représentés, du bloc brut aux percuteurs. L'absence d'enclume doit être relativisée puisque celles qui ont été trouvées ne sont en réalité éloignées des zones d'atelier que de quelques centimètres; sans doute ont-elles été déplacées au terme des activités de taille, geste sans doute fort de signification mais peu interprétable. Les éclats de début de débitage sont fréquents, impliquant le déplacement pour transformation des éclats de fin de débitage. Cette remarque doit toutefois être pondérée en raison de la matière première engagée, à savoir les galets côtiers de silex - de modules réduits qui ne permettent pas toujours l'obtention d'éclats totalement dépourvus de cortex. Dans ces amas qui correspondent à des séquences gestuelles de la part d'un tailleur, la part des esquilles est également beaucoup plus marquée. Plus que la simple obtention de supports, c'est la production d'outils qui semble prioritaire, tout particulièrement pour l'amas 1. Reste la question de la destination réelle de ces produits. En l'absence d'habitat à proximité directe de la zone fouillée, différentes hypothèses peuvent être envisagées. Si un espace domestique proche est plausible, rien n'exclut que ces productions soient diffusées vers des habitats continentaux. La question se pose d'autant que les zones ateliers de Groah Denn 1 ne sont pas les seules connues pour la période du Néolithique récent et pour le Groh Collé notamment : il faut ainsi mentionner le site de Guernic (SaintPierre-Quiberon, Morbihan), zone atelier qui présente les mêmes caractéristiques, à savoir des amas de débitage déconnectés de toutes habitations. Les ateliers plus connus pour le Néolithique final sont traditionnellement des lieux de productions spécialisées, dédiés à la fabrication en série et à grande échelle de diffusion d'un même type d'outil bien particulier tels les lames de haches polies ${ }^{27}$ ou encore les perçoirs (Ponthezières, Saint-

26. Hamon, Gwenaëlle, « Modalités et finalités des dépôts céramiques au Néolithique moyen (4600-3800 av. J.-C.) entre Loire et Normandie ", Du matériel au spirituel, réalités archéologiques et historiques des "dépôts " de la préhistoire à nos jours, $\mathrm{XXIX}^{\mathrm{e}}$ rencontres internationales d'archéologie et d'histoire d'Antibes, 16-18 octobre 2008, Juan-les-Pins, 2009, p. 119-130.

27. Le Roux Charles-Tanguy, L'outillage de pierre polie en métadolérite du type A. Les ateliers de Plussulien (Côtes-d'Armor). Production et diffusion au Néolithique dans la France 
Georges-d'Oléron, Charente-Maritime ${ }^{28}$ ). La chaîne opératoire simple et courte développée sur les ateliers du Groh Collé permet la confection rapide d'un outillage domestique commun. Elle produit en outre une quantité de déchets non négligeable et ce dans un laps de temps très rapide, sans doute de l'ordre de quelques heures. Les amas de débitage témoignent donc d'une production ponctuelle.

\section{Une occupation insulaire}

Bien qu'excentré géographiquement et cerné par la mer, ce site s'intègre pleinement dans la sphère culturelle locale. Aucun particularisme insulaire ne peut être distingué. Si l'on imagine habituellement des populations isolées sur un territoire restreint, l'étude présentée ici démontre qu'il en va tout autrement. Les productions observées tant sur le plan lithique que céramique ne diffèrent en aucun cas de ce qui est connu au même moment au sud du Massif armoricain. Les importations de matière première et/ou de produits finis plaident en faveur d'une zone régulièrement raccordée au domaine continental. Les ateliers de productions lithiques semblent voués à la confection d'un outillage commun dont la destination est très vraisemblablement l'habitat. En l'absence de la découverte de ce dernier, un schéma de diffusion des produits, sur une échelle locale (île-continent), paraît des plus plausibles, d'autant que l'activité s'effectue de façon ponctuelle dans un laps de temps court. Les échanges bien que ponctuels et modérés ne sont donc pas inexistants puisque le gisement prend également place dans un réseau beaucoup plus large, comme en atteste la présence de lames de haches polies importées. Les contacts sont assurés et fréquents avec des populations proches relevant du même groupe culturel mais également avec des populations continentales plus lointaines. Ces dernières inspirant par ailleurs très nettement les tailleurs si l'on en juge l'armature de flèche tranchante de type Sublaines fabriquée localement, signe d'un transfert stylistique et de relations fortes.

Le statut de ce gisement reste quant à lui tout à fait original puisque dépôts et activités de taille se développent ici conjointement. Tous deux relèvent de manifestations ponctuelles (séquences gestuelles courtes) intervenues en des moments différents.

Si à certaines périodes de la préhistoire ou de l'histoire la mer peut apparaître comme une barrière infranchissable, il est désormais certain, à l'appui des découvertes archéologiques récentes, que cela n'a pas toujours été le cas. Pour les périodes préhistoriques et tout particulièrement pour

de l'Ouest et au-delà. Travaux du laboratoire d'anthropologie, préhistoire, protohistoire et quaternaire armoricains, Université de Rennes I, n 43, Rennes, 1999, 1 vol., 244 p., 70 fig. FOUÉRÉ, Pierrick, Les industries en silex..., op. cit.

28. LAPORTE, Luc et al., Des premiers paysans aux premiers métallurgistes sur la façade atlantique (3500-2000 av. J.-C.), Mémoire de la Société de recherches archéologiques de Chauvigny, XXXIII, Chauvigny, 2009, 810 p. 
le Néolithique récent, l'insularité n'apparaît pas comme une contrainte, bien au contraire. Les échanges par voie maritime, bien que ponctuels, sont largement attestés. Il est cependant bien difficile d'en estimer l'ampleur. Les populations implantées sur ces espaces limités de toute part ne diffèrent en rien, si l'on s'en tient à la culture matérielle, des groupes continentaux. À travers l'exemple de Groah Denn 1, mais d'autres contextes équivalents l'évoquent également (Île d'Yeu, Vendée ${ }^{29}$ ), l'insularité ne doit pas être vue comme un isolement mais au contraire comme l'opportunité de déplacements réguliers vers le littoral de la large façade atlantique, facilités par voie maritime; voyages plus lointains (Centre-Ouest) et plus riches (échanges de biens, de style et de traditions techniques) que ceux réalisés par laborieuse et longue voie terrestre.

29. BlanchaRD, Audrey, Le site néolithique récent de la Pointe de la Tranche (île d'Yeu, Vendée), rapport de fouille programmée, SRA de Pays de la Loire, Nantes, 2010, 72 p. 


\section{RÉSUMÉ}

L'archéologie en contexte insulaire n'est pas nouvelle. La richesse archéologique de l'île d'Hoedic est ainsi connue dès la fin du XIX ${ }^{\mathrm{e}}$ siècle. La récente fouille de l'alignement mégalithique de Groah Denn livre néanmoins d'inédites informations sur l'occupation insulaire. Des zones d'ateliers (amas de débitage) dévolus à la production d'outils lithiques ainsi que des dépôts lithique et céramique se développent de part et d'autre de la file de blocs. Corrélés à l'absence d'habitat proche, ces faits ponctuels donnent à ce gisement un statut tout à fait particulier. Ces phénomènes relèvent d'une même phase chronologique qu'est le Néolithique récent, comme en atteste nettement la culture matérielle, mais de moments différents. Le mobilier lithique et céramique mis au jour renvoie ainsi au Néolithique récent régional (IVe millénaire av. J.-C.) et plus précisément au Groh Collé. La production céramique se distingue par la présence de motifs Groh Collé mais également de quelques formes proches du Conguel. Bien qu'en contexte insulaire, les productions découvertes sur le gisement ne diffèrent en rien des productions continentales. Le mobilier recueilli révèle également des échanges avec les côtes proches mais également plus lointaines de matières, de produits ou encore d'idées. Les populations insulaires, au Néolithique récent, ne sont pas isolées mais participent au contraire aux échanges, dépassant largement les limites imposées par la mer.

\section{ABSTRACT}

Archaeology in an island context is no new thing. For instance, the archaeological wealth of Hoédic has been known since the end of the 19th century. The recent excavation of the megalithic alignment of Groat Denn gives new information on the occupation of the island. Zones of "workshops" (marked by heaps of debris) producing stone tools, as well as lithic and ceramic deposits are to be found on either side of the alignment. Together with the absence of nearby domestic settlements, this gives the site a status of its own. These facts belong to the same period of time, namely the late Neolithic, as is clearly evidenced by the material culture from different moments. So, the lithic and ceramic items brought to light send us back to the late Neolithic ( $4^{\text {th }}$ millennium B.C.) in the district, more precisely at Groh Collé. The ceramic production stands out, with the presence of Groh Collé motifs as well as some shapes close to Conguel. Though found in an island context, those productions are in no way different from continental ones. The items found there also reveal exchanges of materials, products, and ideas, with nearby shores as well as with more distant ones. The island populations, in the late Neolithic, are not isolated; on the contrary, they take part in exchanges that widely exceed the limits imposed by the sea. 